\title{
Review of: "Microchannelled alkylated chitosan sponge to treat noncompressible hemorrhages and facilitate wound healing"
}

\author{
yakai feng $^{1}$ \\ 1 Tianjin University
}

Potential competing interests: The author(s) declared that no potential competing interests exist.

Massive blood loss often causes significant mortality in civilian and battlefield settings. Traditional hemostats show limited anti-bleeding ability due to the lack of proper design on physical structure (especially pore structure) and of active modification. Moreover, after hemostasis, they need to be removed from the wound bed because of their inhibition effect on tissue repair, leading to secondary bleeding and the pain of patients. Therefore, developing a shape-memory hemostat left in the injury site and used in directly guided in situ tissue regeneration is critical need and more practical for clinical application. Additionally, the application of such hemostat will reduce patient discomfort, simplify treatment procedures, and potentially decrease healthcare costs.

At present, the pore structure of most clinically used hemostatic materials is derived from ice crystal, which is lack of controllability, connectivity and gradient. The hemostatic material prepared by Du et al. possess a gradient interconnected channel structure, the macro channels can promote the rapid blood penetration, and the micropore within the matrix can accelerate the local blood concentration and coagulation. In addition, alkylation activity modification further improved the hemostatic and antibacterial properties. More importantly, the introduction of microchannels can guide tissue in situ regeneration and accelerate the ingrowth of tissues, cells and blood vessels. In this way, hemostatic materials can be left at the wound site to guide in situ tissue regeneration. This microchannelled alkylated chitosan sponge had great clinical translational potential in applications of lethal noncompressible hemorrhage and penetrating wound healing. The design of hemostatic active material with gradient pore structure may change the current concept that hemostatic materials need to be taken out, and provide a new concept and strategy for the design and construction of hemostatic materials in the future. 